\title{
Thiol-Ene Click Microcontact Printing of Gold Nanoparticles onto Silicon Surfaces
}

\begin{tabular}{|r|l|}
\hline Journal: & Canadian Journal of Chemistry \\
\hline Manuscript ID & Cjc-2017-0321.R1 \\
\hline Manuscript Type: & Article \\
\hline Date Submitted by the Author: & 31 -Aug-2017 \\
\hline Complete List of Authors: & $\begin{array}{l}\text { Platnich, Casey; University of Calgary, Chemistry; McGill University, } \\
\text { Chemistry } \\
\text { Banerjee, Abhinandan; University of Calgary, Chemistry } \\
\text { Ozhukil Kollath, Vinayaraj; University of Calgary, Chemical \& Petroleum } \\
\text { Engineering } \\
\text { Karan, Kunal; University of Calgary, Chemical \& Petroleum Engineering } \\
\text { Trudel, Simon; University of Calgary, Chemistry }\end{array}$ \\
\hline $\begin{array}{r}\text { Is the invited manuscript for } \\
\text { consideration in a Special } \\
\text { Issue?: }\end{array}$ & SFU \\
\hline Keyword: & Click chemistry, microcontact printing, surface chemistry \\
\hline \multicolumn{2}{|c}{} \\
\hline \multicolumn{2}{|c|}{} \\
\hline
\end{tabular}

SCHOLARONE ${ }^{\text {IM }}$

Manuscripts 


\title{
Thiol-Ene Click Microcontact Printing of Gold Nanoparticles onto Silicon Surfaces
}

\author{
Casey M. Platnich, Abhinandan Banerjee, Vinayaraj Ozhukil Kollath, Kunal Karan, and \\ Simon Trudel*
}

\begin{abstract}
We report a novel process to selectively pattern nanomaterials, specifically gold nanoparticles, onto a silicon surface through "click" chemistry, to consistently and efficiently join together small units through a quick and simple reaction. We employed the UV-initiated thiol-ene reaction, which is used in tandem with microcontact printing. Dithiolcapped nanoparticles were used as a printing ink and were grafted onto ene-terminated $\mathrm{Si}(100)$ wafers by pressing a nanoparticle-impregnated poly(dimethylsiloxane) stamp, while irradiating with ultraviolet light to activate a radical initiator. The resulting structures were characterized using scanning electron microscopy.
\end{abstract}

Key words: click chemistry, microcontact printing, surface chemistry.

Résumé :

Mots clés : mots cles.

\section{Introduction}

Nanoparticles (NPs) anchored to semiconducting surfaces can find applications in sensing, ${ }^{1}$ catalysis, ${ }^{2}$ or spectroscopy. ${ }^{3}$ It may however be challenging to deliberately place nanoparticles onto specific areas to create patterns without resorting to expensive or low-throughput top-down methods such as electronbeam lithography. In this work, we show how a combination of click chemistry ${ }^{4}$ and soft lithographic micro-contact printing $(\mu \mathrm{CP})^{5}$ can anchor gold nanoparticles (Au NPs) onto silicon surfaces.

In order to selectively pattern nanoparticles into precise architectures, microcontact printing $(\mu \mathrm{CP})$ was employed. Microcontact printing is a type of soft lithography that uses polymer stamps, usually made from poly(dimethylsiloxane) (PDMS). ${ }^{5}$ Microcontact printing functions as a classical stamp-and-ink method: a PDMS stamp is coated with an ink solution containing the desired molecule for printing, dried and then brought into conformal contact with the substrate. In this way, the reaction only takes place in the areas of direct contact between the stamp and the substrate, allowing for positional

Received . Revision received . Accepted . Revision accepted .

Casey M. Platnich. Department of Chemistry, University of Calgary, 2500 University Dr NW, Calgary, AB, Canada T2N 1N4; now at Department of Chemistry, McGill University, 801 Sherbrooke St. West, Montréal, QC, Canada H3A 0B8

Abhinandan Banerjee. Department of Chemistry and Institute for Quantum Science and Technology, University of Calgary, 2500 University Dr NW, Calgary, AB, Canada T2N 1N4

Vinayaraj Ozhukil Kollath. Department of Chemical \& Petroleum Engineering, Schulich School of Engineering, University of Calgary, 2500 University Dr NW, Calgary, AB, Canada T2N 1N4 Kunal Karan. Department of Chemical \& Petroleum Engineering, Schulich School of Engineering, University of Calgary, 2500 University Dr NW, Calgary, AB, Canada T2N 1N4

Simon Trudel*. Department of Chemistry and Institute for Quantum Science and Technology, University of Calgary, 2500 University Dr NW, Calgary, AB, Canada T2N 1N4; * Corresponding author: trudels@ucalgary.ca control over the chemical functionalization. The seminal example is the patterning of gold surfaces with an alkanethiol. ${ }^{5}$ By forming the stamp on a master of a certain design, the same pattern can be easily reproduced onto the substrate. An important advantage of microcontact printing lies in the high concentration of ink in the areas of contact, allowing reactions to proceed quickly while using only small amounts of ink to obtain high-resolution patterning. In addition to patterning molecules, microcontact printing has been used to deliver nanoparticles onto surfaces. Recent examples include the deposition of $\mathrm{TiO}_{2}$ NPs on glass and Si surfaces, ${ }^{6}$ Au NPs on solid substrates ${ }^{7}$, $\mathrm{Au}^{8}$ and $\mathrm{Ag}^{9}$ on flexible polyethylene terephthalate substrates, diamond NPs on poly(methyl methacrylate)-coated Si substartes, ${ }^{10}$ and polymer particles onto planar Si substrates. ${ }^{11}$ However, in all these methods, the NPs are not covalently bound to the substrate, which can lead to durability issues. In this work, we take advantage of click chemistry between the NP ink and substrate surface used for $\mu \mathrm{CP}$ to covalently tether $\mathrm{Au}$ NPs to a functionalized silicon surface.

Click chemistry denotes a branch of chemical reactions that react very selectively and regiospecifically under mild conditions (low temperatures, aqueous solutions, in air). ${ }^{4}$ These reactions typically give very high yields and can be performed with readily available reagents and easily handled solvents, making them an area of particular interest for many industrial applications. Although some click reactions have been extensively investigated, such as the Huisgen 1,3-cycloaddition, other click reactions remain relatively unexplored for the purposes of self-assembly. ${ }^{12}$ Click chemistry has been used to covalently bind several types of chemical entities to surfaces, including enzymes, ${ }^{13}$ self-assembled monolayers ${ }^{14-16}$ including photochemically initiated thiol-ene and thiol-yne reactions, ${ }^{17}$ carbohydrates, ${ }^{18}$ and rewritable polymer brush micropatterns. ${ }^{19}$ There are few examples where click chemistry was used to tether NPs in conjunction with $\mu \mathrm{CP}{ }^{20-22}$ Huisgen 1,3-cycloaddition was used to pattern $\mathrm{Au} \mathrm{NPs},{ }^{22}$ while bromine-terminated Co nanoparticles were attached to aminopropyl-terminated Si surfaces. ${ }^{20,21}$

Here we use thiol-ene click chemistry, i.e. the addition of 
Thermal surface hydrosylilation

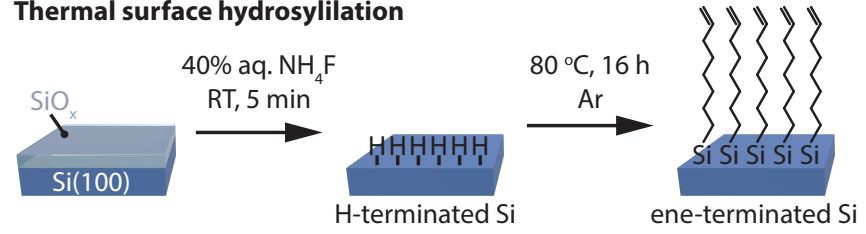

Thiol-ene click reaction

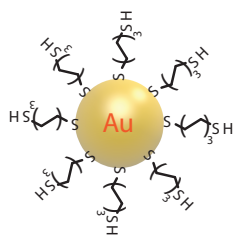
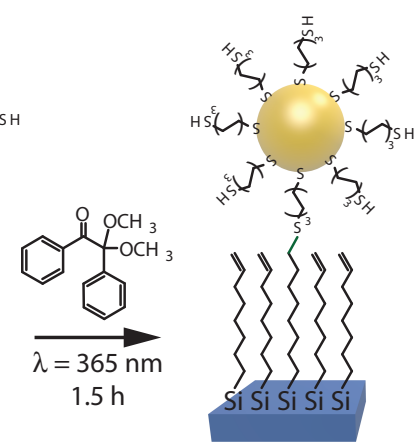

Fig. 1. Reaction scheme. Top: hyrosylilation reaction of H-terminated $\mathrm{Si}(100)$ to form a self-assembled monolayer of 1,7-octadiene. Bottom: Thiol-ene reaction attaching dithiol-coated gold nanoparticles to alkene-terminated silicon wafer.

a thiol functional group to an alkene, to bind gold nanoparticles onto silicon surfaces (see Fig. 1). ${ }^{23}$ In order to harness the thiol-ene reaction to attach nanostructures to the ene-terminated silicon surface, gold nanoparticles were synthesized and stabilized with dithiol molecules, resulting in functionalization with a thiol group. ${ }^{24}$ The thiol-ene reaction proceeds via a UV-initiated radical mechanism with the aid of a photoinitiator, such 2,2-dimethoxy-2-phenylacetophenone (DMPA). ${ }^{23}$ Of particular interest to surface patterning, this thiol-ene reaction benefits from its ability to proceed in the absence of solvent. ${ }^{17}$ Silicon(100) is widely used as a semiconducting substrate in microelectronics. By wet etching with ammonium fluoride, the native oxide layer can be easily removed from the silicon, resulting in a hydrogen-terminated silicon, which can then be reacted by thermal initiation with an alkene in a simple addition reaction. ${ }^{25}$ This protocol gives a densely packed monolayer that both provides protection to the surface against oxidation and presents a double bond for further reaction (in this case, with thiols). This type of surface modification can be done quickly and easily and the linkages formed should be robust, due to the strong silicon-carbon bonds that result. This system is also versatile in that subsequent functionalization of the gold nanoparticles is possible through further thiolene chemistry, leaving the bare substrate unmodified.

\section{Experimental Details}

Methods and Materials. All chemicals were purchased from Sigma Aldrich or VWR and were used as received unless otherwise indicated. Ultrapure water with a specific resistivity of at least $18 \mathrm{M} \Omega \mathrm{cm}^{-1}$ was produced using a Purelab deionization system and was used for all procedures unless otherwise stated. For the light-induced thiol-ene chemistry, a high-power $365 \mathrm{~nm}$ UV lamp was used and was brought within $5 \mathrm{~cm}$ of the surface. Air-free modification of wafers was per- formed using standard Schlenk techniques under argon and the 1,7-octadiene was degassed by three freeze-pump-thaw cycles before use and stored under argon.

Surface Modification. Silicon(100) wafers were cut into 1 $\times 1 \mathrm{~cm}$ sized squares. These wafers were then cleaned by rinsing with acetone, ethanol, methanol, and water and blown dry in a stream of argon. The wafers were then etched in a $40 \%$ aqueous solution of ammonium fluoride $\left(\mathrm{NH}_{4} \mathrm{~F}\right)$ for five minutes to remove the native oxide layer and then rinsed with copious amounts of water and blown dry under argon. Etching of the wafer is confirmed by observing the water contact angle; the character of the wafer should changes from hydrophilic to hydrophobic with the removal of the native oxide $\left(\mathrm{SiO}_{x}\right)$ layer and the change was clearly visible.

Once etched, the wafers were placed in a Schlenk flask, the atmosphere pumped off and the vessel sealed under argon. Approximately $5 \mathrm{~mL}$ neat, degassed 1,7-octadiene was then added to the vessel. The vessel was then heated to $80{ }^{\circ} \mathrm{C}$ for $16 \mathrm{~h}$ using a sand bath. ${ }^{25}$ Following the reaction, the wafers were exposed to air and cleaned thoroughly by rinsing with hexanes, methanol and water, then dried with argon. The wafers were stored in the dark in a petri dish to avoid contamination and used promptly within ten days of modification.

Nanoparticle Synthesis. The synthesis of gold nanoparticles was adapted from Hostetler et al. ${ }^{24}$. A solution of $1.5 \mathrm{~g}$ (2.5 equivalents) tetraoctylammonium bromide (TOAB) in 80 $\mathrm{mL}$ toluene was prepared. To this vigorously stirring solution, $0.31 \mathrm{~g}$ ( 1 equivalent) tetrachloroauric acid $\left(\mathrm{HAuCl}_{4} \cdot \mathrm{xH}_{2} \mathrm{O}\right)$ in $25 \mathrm{~mL}$ water was added. The yellow $\mathrm{HAuCl}_{4} \cdot \mathrm{xH}_{2} \mathrm{O}$ solution immediately cleared and the toluene phase become orangebrown as the $\mathrm{AuCl}_{4}^{-}$ions moved into this organic layer. The organic phase was then isolated and $14.2 \mu \mathrm{L}(0.083$ equivalents) of 1,6-hexanedithiol was added. This solution was then stirred for 10 minutes and the orange-brown colour persisted. The reaction solution was then reduced using a solution of $\mathrm{NaBH}_{4}$ (0.38 g, 10 equivalents) in $25 \mathrm{~mL}$ water, which was added drop-wise over a period of 15 minutes. The very dark organic phase was then stirred for $3.5 \mathrm{~h}$. At this point, the organic phase was isolated and the solvent was removed on a rotary evaporator (this step should not exceed $50{ }^{\circ} \mathrm{C}$ to prevent any product decomposition). The black product was then suspended in $30 \mathrm{~mL}$ ethanol, sonicated for 1 minute to dissolve by-products and collected on a glass filtration frit. The product was then washed with at least $80 \mathrm{~mL}$ of ethanol and $150 \mathrm{~mL}$ of acetone and vacuum dried on the filtration frit. The yield for this process is approximately $120 \mathrm{mg}$ gold nanoparticles.

Thiol-ene Reaction. The thiol-ene procedure was adapted from Schulz et al. ${ }^{25}$ For thiol-ene reactions carried out in the absence of a stamp, a solution of gold nanoparticles $(0.010$ g) and DMPA $(0.2561 \mathrm{~g})$ in $1 \mathrm{~mL}$ solvent was prepared. The solvent used was either chlorobenzene or ethanol. This solution was sonicated for 5 minutes to ensure dispersion of the nanoparticles and dissolution of the photoinitiator. A clean, dry, alkene-terminated silicon wafer was then placed face-up in a small Petri dish and fully covered with the nanoparticle solution. This substrate was then irradiated for $1.5 \mathrm{~h}$ with $365 \mathrm{~nm}$ UV light to carry out out the reaction over the whole sample. To ensure the nanoparticles are clearly attached to be substrate, the wafer was cleaned after the reaction by rinsing with either chlorobenzene or ethanol (whichever solvent was used origi- 
nally) and sonicated for 1 minute in this solvent. The wafer was then rinsed with hexanes and dichloromethane to remove any excess solution and dried with argon. Modified wafers appear dark grey in colour and matte (Fig. S2).

Microcontact Printing. Flat PDMS stamps were prepared by combining poly(dimethylsiloxane) and curing agent of the "Sylgard 184" elastomer kit in a ratio of 10:1 (by volume) and pouring them onto an unmodified silicon wafer that had been washed with acetone, ethanol, methanol, and water and dried in argon. It is important that bubbles be avoided to prevent imperfections from forming in the stamp. This stamp is then cured in an oven at $70^{\circ} \mathrm{C}$ for $3 \mathrm{~h}$. Patterned stamps were made in the same way, except a compact disk, cleaned with methanol and water and dried in argon, is used as the master instead of the silicon wafer. Stamps of any size can then be cut from the main slab of polymer. The stamps were cleaned by sonicating them for 10 minutes in ethanol and blown dry in a stream of argon.

Stamps were soaked in a gold nanoparticle "ink" consisting of gold nanoparticles $(0.010 \mathrm{~g})$ and DMPA $(0.2561 \mathrm{~g})$ in $1 \mathrm{~mL}$ of either ethanol or chlorobenzene for 10 minutes. The stamps were then blown dry in a stream of argon. The stamp was then brought into contact with the clean, dry, alkene-terminated silicon wafer and the wafer was irradiated for $1.5 \mathrm{hr}$ with 365 nm UV light through the PDMS stamp. After the reaction, the stamp was carefully removed; the wafer was cleaned with the original solvent and sonicated for 1 minute. The wafer was then rinsed with hexanes and dichloromethane to remove any excess solution and dried with argon.

Physical Characterization. UV-vis extinction spectra were recorded on a Cary 5 spectrometer. Fourier-transform infrared (FTIR) spectra of gold nanoparticles in a $\mathrm{KBr}$ disk were recorded on a Thermo Nicolet Nexus 470 spectrometer operating at $4 \mathrm{~cm}^{-1}$ resolution. Scanning electron microscopy was carried out with a Zeiss $\Sigma$ igma VP field-emission microscope. Transmission electron microscopy was carried out using a Hitachi Hitachi H7650 microscope operated at $100 \mathrm{kV}$. Samples were prepared by drop-casting a suspension of the particles onto a carbon-coated copper mesh grid (Ted Pella). The contact angles were determined using sessile drop method in an Advanced Goniometer (Model: 500F4, ramé-hart co., USA) system equipped with a video camera and a light source. Image analysis for contact angle and surface energy calculation were completed using the software (DROPimage V2.7.03) provided by the manufacturer. A high precision micro syringe was used to produce repeatable volumes of the liquid droplet. To assess hydrophobic or hydrophilic characteristics, MilliQ water $\left(18.2 \mathrm{M} \Omega\right.$ at $\left.25^{\circ} \mathrm{C}\right)$ was used as the liquid. Contact angles of both left and right side of the sessile drop was calculated by the software from 10 measurements at $1 \mathrm{sec}$ interval, after dispensing each drop. Measurements were repeated at different locations of the sample. Atomic force microscopy imaging (N9410S, 5500 AFM, Keysight Technologies) was performed in acoustic alternating contact mode, using a $\mathrm{Si}_{3} \mathrm{~N}_{4}$ soft tapping type AFM probe (HQ:NSC14/Al BS, $\mu$ Masch) with a resonance frequency of $\mathrm{ca} .136 \mathrm{kHz}$. Data acquisition was at 256 pts.line ${ }^{-1}$ with a scan speed of 0.3 line $\cdot \mathrm{s}^{-1}$. Scan size was varied based on the feature sizes.

\section{Results and Discussion}

Silicon(100) wafers were first etched in a $40 \%$ aqueous solution of ammonium fluoride $\left(\mathrm{NH}_{4} \mathrm{~F}\right)$ to remove the native oxide layer. ${ }^{26}$ By observing the water contact angle formed between the water and the substrate it was clear that etching had in fact occurred; the oxide layer exhibits hydrophilic character $\left(\theta=37.3^{\circ}\right)$ whereas the hydrogen-terminated silicon shows hydrophobic behaviour $\left(\theta=78.3^{\circ}\right)$ (Fig. S1). This shift in behaviour is clearly visible and allows for the easy determination of whether or not the etching has been successful. Even with as little as five minutes of etching, the surface appears hydrophobic, with water forming droplets with a high contact angle on the surface of the silicon.

Once etched, the wafers were placed in a reaction vessel and the atmosphere was pumped off for air-free reaction with degassed diene. The surface modification is done in a completely air-free environment to prevent an oxide layer from reforming. A long, linear diene is required to form densely packed monolayers, which protect the surface from oxidation and allow for greater opportunity for reaction with thiols. That being said, an extremely long carbon chain this would increase the likelihood of having both ends of the diene attach to the surface, which is undesirable as this leads to less alkene ends being available for reaction. If shorter chains are used, steric considerations prohibit this from occurring. ${ }^{27}$ For these reasons, 1,7-octadiene is a reasonable choice for forming this monolayer. Once the monolayer is formed, the wafers can be exposed to air, as the thick alkene monolayer presents oxidation from rapidly occurring at the surface.

The synthesis of gold nanoparticles followed a 2-phase synthesis of Murray and coworkers. ${ }^{24}$ This type of synthesis was chosen as it allowed for a reasonable yield to be obtained and the nanoparticles can be isolated in their stable, solid form, meaning that they can easily be handled and can be stored at room temperature under no special conditions. ${ }^{28}$ Because the carbon chain is not exceptionally long, steric restraints likely prohibit both ends of the thiol attaching to the gold surface, leaving one group available for the click reaction. In addition, by using a linear chain dithiol, we hope to achieve reasonably good density of thiol on the gold nanoparticle to increase its probability of successfully binding to the surface. ${ }^{29}$ Dithiol was used as the capping ligand in a ratio of 1:12 dithiol to gold. This ratio was found to result in the largest particle size. ${ }^{24}$ Particles were made to the maximum size in order to simplify the task of imaging these nanostructures and in an attempt to have many thiols available for bonding at the surface.

The synthesis of the dithiol-coated gold was verified by suspending a sample of the nanoparticles in toluene and performing UV-Visible spectroscopy. The resulting spectrum is shown in Figure $2 \mathrm{a}$ and shows a peak in extinction at a wavelength of $520 \mathrm{~nm}$ (Figure 2a). This peak corresponds to the surface plasmon resonance of the nanoscaled gold, and corresponds to the literature values for thiol-coated gold nanoparticles of a similar size. ${ }^{24}$ The reaction product was also imaged using transmission electron microscopy (TEM), confirming the synthesis of gold nanoparticles (Figure 2b). The particles range in size and are clearly not monodisperse, but for the purposes of verifying the validity of the thiol-ene reaction and microcontact printing procedures, this was deemed unimportant. The infrared spectrum of the nanoparticles is shown in Figure 2c. The peaks are 

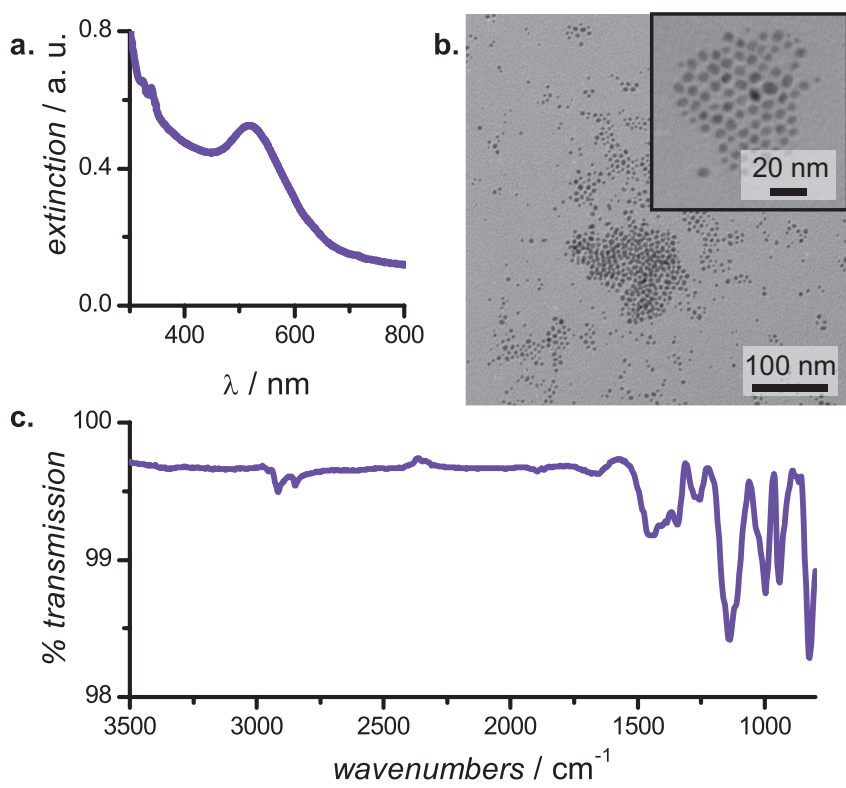

Fig. 2. Characterization of Au NPs. a. UV-Visible spectrum of dithiol-coated gold nanoparticles in toluene. $\lambda_{\max }=520 \mathrm{~nm}$. b. TEM images of the dithiol-coated Au NPs. c. Infrared spectrum of dithiol-coated Au NPs (KBr disk).

as expected for an alklane dithiol; ${ }^{30}$ however we note the $S$ $\mathrm{H}$ stretch is not clearly visible. It is unclear whether this is a result of poor oscillator strength, low concentration, or bidentate binding of the dithiol to the gold surface. The likelihood of larger clusters is also relatively high because of the use of dithiol; because the ligand contains a thiol functional group on each end, it is possible for the ligand to bind together two particles if the thiol at each end of the molecule each binds to a separate gold particle.

To test the validity of the method a thiol-ene reaction was attempted in the absence of a stamp and under blanket UV irradiation. To do this, solutions of gold nanoparticles and DMPA in either chlorobenzene or ethanol were created. The reaction's success can be confirmed visually: after reaction, the wafers appear dark grey in colour and matte (see Figure S2). SEM images also confirm the success of this reaction, both in ethanol and in chlorobenzene (Figure 3). Even after several rinsing with different solvents, the gold nanoparticles remain attached to the surface and can be easily seen by SEM, confirming they are indeed attached to the surface. While nanoparticles are indeed covering the surface, the coating appears thick, as no mechanism limits the agglomeration of nanoparticle from solution. In order to confirm that the reaction was proceeding via the suggested photoinitiated mechanism, a control was performed: the same reaction was carried out but without the application of UV light (the reaction was carried out inside a dark box to ensure no UV radiation). Visually, this wafer appeared unmodified and SEM confirmed that no nanoparticles had been attached to the silicon (see Fig. S3). This suggested that the reaction does in fact proceed by the UV-initiated thiol-ene reaction proposed and that its mechanism is radical in nature, aided by the photoinitiator DMPA.

Once the reaction without a stamp was successfully achieved, microcontact printing was attempted using PDMS

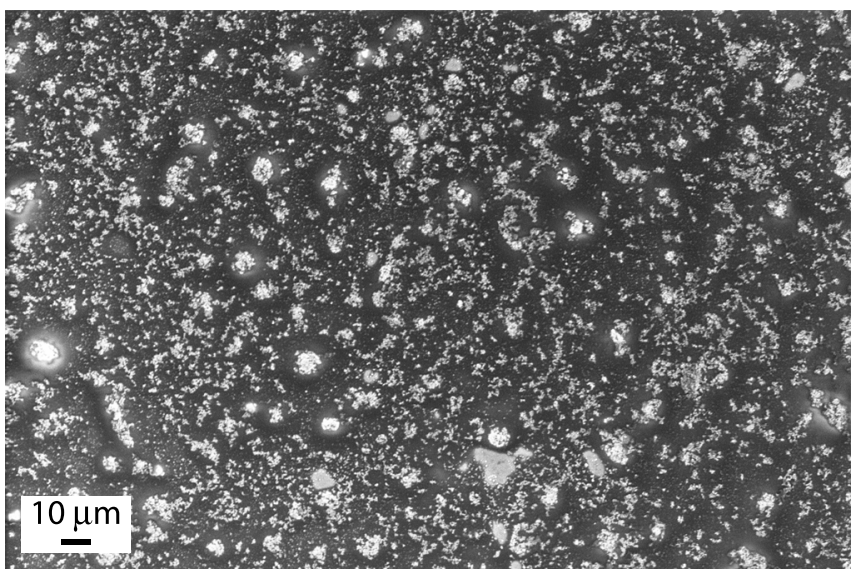

Fig. 3. SEM image of gold nanoparticle ink (10mg NPs, $0.25 \mathrm{~g}$ DMPA, $1 \mathrm{~mL}$ EtOH) reacted via thiol-ene onto alkene-modified silicon wafer in reaction with no stamp and under blanket UV irradiation.

stamps (Figure 4). At first, flat stamps were used (Figure 5a and c). These surfaces were then characterized by SEM imaging. Unlike the solution reaction (Figure 3) a thin coating of NPs is seen on the surface, indicating the process appears to be limited to the deposition of a monolayer of NPs during stamping. The coating is uniform, though not dense. Using a $\mathrm{CD}$ as the master, patterned PDMS stamps with micron-wide indentations were produced. One reason why ethanol may be a preferable solvent for this process becomes important here; chlorobenzene causes PDMS to swell slightly, which can cause deformations in the pattern of the stamp, which then results in defects in the final pattern on the substrate. The swelling of PDMS occurs to some degree with most solvents, but is relatively minor with ethanol, making it a better solvent for this application. ${ }^{31}$ The SEM images show, however, incomplete lines, indicating that the density of nanoparticles was not sufficient for creating dense lines (Figure 5b and d), consistent with the results with the flat stamps above. The reasons for this irregularity in the pattern are likely multifold. Firstly, no pressure was applied to the stamp, therefore it is likely that the stamp was not brought into perfectly even contact with the surface, resulting in good patterning in some areas and sparse in others. Secondly, the concentration of the ink solution has not been optimized, therefore it is possible that there was simply not a high enough concentration of nanoparticles in the solution to provide good pattern density. Finally, a prolonged irradiation time may yield to denser coverage.

Finally, to address whether single or multiple layers of NPs were deposited in this process, atomic force microscopy (AFM) was carried out. As can be seen in Figure 6, two different types of features are observed in the micrographs, namely features with heights of roughly 130 and $30 \mathrm{~nm}$. The first may be related to cluster of Au NPs (which we deem to be due to aggregation in solution), while the latter are congruent with single nanoparticles. When combined with SEM images (Figure 5) which do not show full coverage, it seems that submonolayer single-particle coverage is likely. 


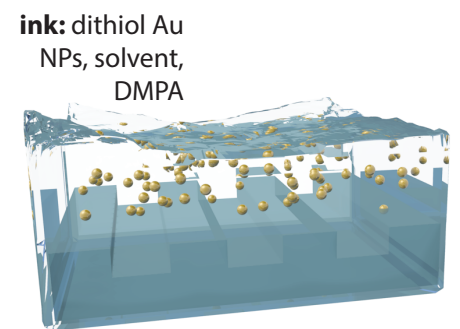

1. PDMS stamp soaked in ink

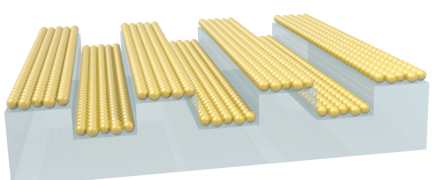

2. ink dries

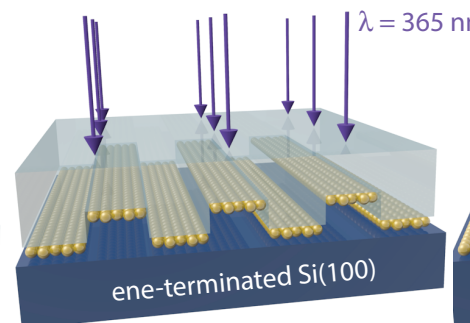

3. bring stamp into contact while irradiating with UV

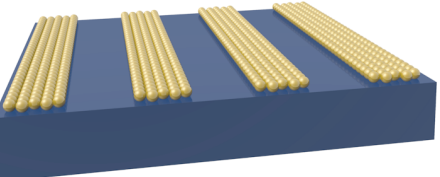

4. final pattern

Fig. 4. Microcontact printing process. Gold nanoparticles bind only in areas of direct contact.
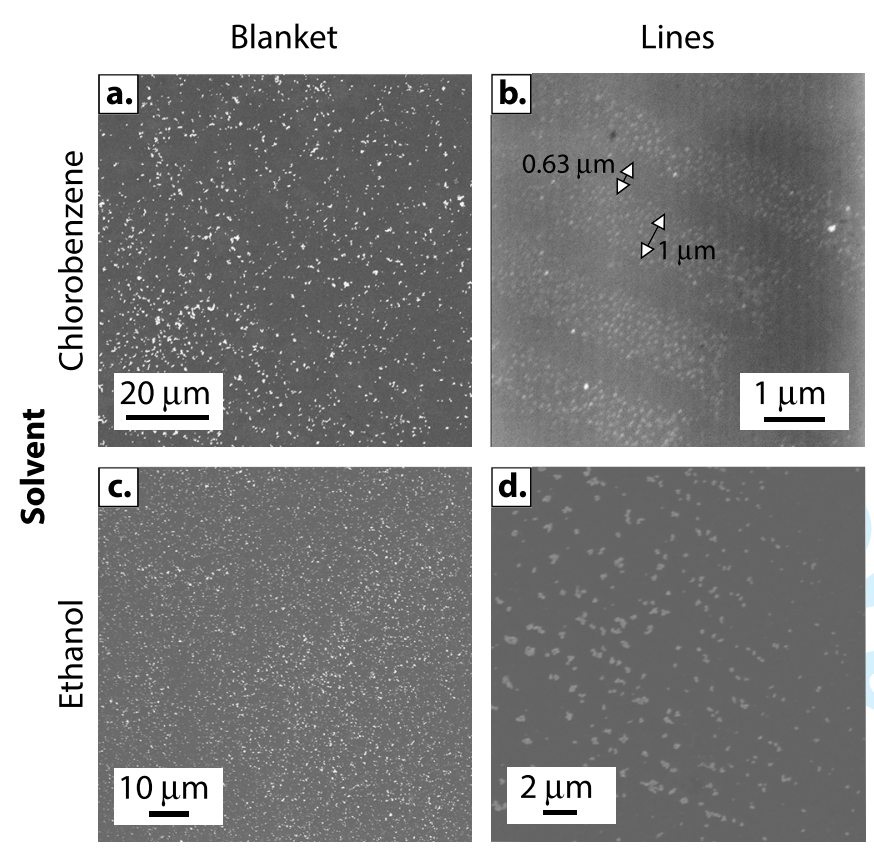

Fig. 5. SEM images of an example of patterns produced using a PDMS stamp made with CD as master. Sample shown was prepared using chlorobenzene as the solvent. Micron wide lines can be seen, as labelled in the image on the right, indicating the pattern formed by the CD. SEM images of ethanol samples. Left side shows results of reaction without a stamp, right side shows results of $\mathrm{CD}$ stamp microcontact printing.

\section{Conclusions}

Microcontact printing, paired with the thiol-ene reaction appears to be a valid process to selectively attach nanostructures in distinct patterns on silicon. Gold nanoparticles coated with dithiol ligands were successfully attached to an alkene modified silicon surface, both with and without the application of a PDMS stamp. Micron-resolution lines were successfully produced using this method, although this process needs tuning in order to optimize the quality of the patterns obtained.Optimizing this proof of concept to allow for dense nanoparticle architectures would allow for the low-cost manufacturing of microelectronics and other patterning applications, including sensing applications. This process would allow for this to be carried out with relatively available reagents and under mild reaction conditions, making it a convenient method
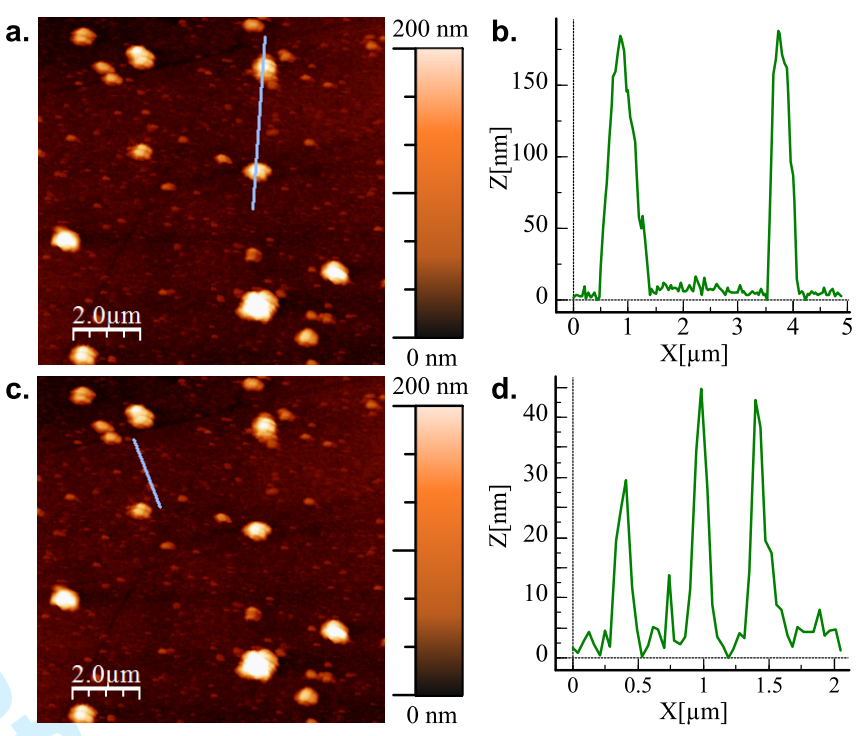

Fig. 6. Atomic force micrograph showing that particles with a ca. $130 \mathrm{~nm}$ height (a-b) and 30-40 nm height (c-d). Scan size is 9.8 $\times 9.8 \mu \mathrm{m}^{2}$.

for rapid prototyping. The potential to reuse PDMS stamps made from a single master would also make this process efficient and economical.

\section{Acknowledgments}

This work was funded by the Natural Sciences and Engineering Research Council (NSERC) of Canada through a Discovery grant, the University of Calgary with an Eyes High postdoctoral scholarship (ARB and VOK) and a URGC grant, and used infrastructure funded by the Canadian foundation for Innovation (CFI) through a John R. Evans Leaders Fund grant.

\section{References}

1. Anker, J. N.; Hall, W. P.; Lyandres, O.; Shah, N. C.; Zhao, J.; Van Duyne, R. P. Nat. Mater. 2008, 7, 442-453.

2. Campbell, C. T.; Sellers, J. R. V. Faraday Discuss. 2013, $162,9-30$. 
3. Hicks, E. M.; Lyandres, O.; Hall, W. P.; Zou, S.; Glucksberg, M. R.; Van Duyne, R. P. J. Phys. Chem. C 2007, $111,4116-4124$.

4. Kolb, H. C.; Finn, M. G.; Sharpless, K. B. Angew. Chem. Int. Ed. 2001, 40, 2004-2021.

5. Gates, B. D.; Xu, Q.; Stewart, M.; Ryan, D.; Willson, C. G.; Whitesides, G. M. Chem. Rev. 2005, 105, 1171-1196.

6. Lamping, S.; Ravoo, B. J. J. Mater. Chem. C 2017, 5, 5882-5886.

7. Santhanam, V.; Andres, R. P. Nano Letters 2004, 4, 4144.

8. Han, S.-T.; Zhou, Y.; Xu, Z.-X.; Huang, L.-B.; Yang, X.B.; Roy, V. A. L. Advanced Materials 2012, 24, 35563561 .

9. Xin, Z.; Liu, Y.; Li, X.; Liu, S.; Fang, Y.; Deng, Y.; Bao, C.; Li, L. Materials Research Express 2017, 4, 015021.

10. Zhuang, H.; Song, B.; Staedler, T.; Jiang, X. Langmuir 2011, 27, 11981-11989, PMID: 21866927.

11. Sieb, N. R.; Gates, B. D. Advanced Materials 2008, 20, 1376-1380.

12. Moses, J. E.; Moorhouse, A. D. Chem. Soc. Rev. 2007, $36,1249-1262$.

13. Buhl, M.; Vonhören, B.; Ravoo, B. J. Bioconjugate Chemistry 2015, 26, 1017-1020, PMID: 26030726.

14. Wang, J.; Wu, F.; Watkinson, M.; Zhu, J.; Krause, S. Langmuir 2015, 31, 9646-9654, PMID: 26274063.

15. Mehlich, J.; Ravoo, B. J. Org. Biomol. Chem. 2011, 9, 4108-4115.

16. Rozkiewicz, D. I.; Jańczewski, D.; Verboom, W.; Ravoo, B. J.; Reinhoudt, D. N. Angewandte Chemie International Edition 2006, 45, 5292-5296.

17. Wendeln, C.; Rinnen, S.; Schulz, C.; Arlinghaus, H. F.; Ravoo, B. J. Langmuir 2010, 26, 15966-15971.

18. Michel, O.; Ravoo, B. J. Langmuir 2008, 24, 1211612118, PMID: 18837529.

19. Roling, O.; De Bruycker, K.; Vonhören, B.; Stricker, L.; Körsgen, M.; Arlinghaus, H. F.; Ravoo, B. J.; Du Prez, F. E. Angewandte Chemie International Edition 2015, 54, 13126-13129.

20. Bae, S.-S.; Lim, D.; Park, J.-I.; Cheon, J.; Jeon, I.; Kim, S. Applied Physics A 2005, 80, 1305-1310.

21. Bae, S.-S.; Lim, D. K.; Park, J.-I.; Lee, W.-R.; Cheon, J.; Kim, S. The Journal of Physical Chemistry B 2004, 108, 2575-2579.
22. Ina Rianasari, I.; de Jong, M. P.; Huskens, J.; van der Wiel, W. G. Int. J. Mol. Sci. 2013, 14, 3705-3717.

23. Hoyle, C.; Bowman, C. Angew. Chem. Int. Ed. 2010, 49, 1540-1573.

24. Hostetler, M. J.; Wingate, J. E.; Zhong, C.-J.; Harris, J. E.; Vachet, R. W.; Clark, M. R.; Londono, J. D.; Green, S. J.; Stokes, J. J.; Wignall, G. D.; Glish, G. L.; Porter, M. D.; Evans, N. D.; Murray, R. W. Langmuir 1998, 14, 17-30.

25. Schulz, C.; Nowak, S.; Frohlich, R.; Ravoo, B. J. Small 2012, 8, 569-577.

26. Bae, S.-E.; Oh, M.-K.; Min, N.-K.; Paek, S.-H.; Hong, S.-I.; Lee, C.-W. J. Bull. Korean Chem. Soc. 2004, $25,1822-1828$.

27. Campos, M. A. C.; Paulusse, J. M. J.; Zuilhof, H. Chem. Commun. 2010, 46, 5512-5514.

28. Brust, M.; Walker, M.; Bethell, D.; Schiffrin, D. J.; Whyman, R. J. Chem. Soc., Chem. Commun. 1994, 801-802.

29. Sieval, A. B.; van den Hout, B.; Zuilhof, H.; Sudhölter, E. J. R. Langmuir 2001, 17, 2172-2181.

30. Himmelhaus, M.; Eisert, F.; Buck, M.; Grunze, M. J. Phys. Chem. B 2000, 104, 576-584.

31. Lee, J. N.; Park, C.; Whitesides, G. M. Anal. Chem. 2003, 75, 6544-6554. 


\section{Electronic Supplementary Information: Thiol-Ene Click Microcontact Printing of Gold Nanoparticles onto Silicon Surfaces}

Casey M. Platnich, ${ }^{a, b}$ Abhinandan Banerjee, ${ }^{a}$ Vinayaraj Ozhukil Kollath ${ }^{c}$ Kunal Karan, ${ }^{c}$ and Simon Trudel $^{* a}$

\footnotetext{
${ }^{a}$ Department of Chemistry and Institute for Quantum Science and Technology, University of Calgary, 2500 University Drive NW, Calgary, AB, Canada, T2N 1N4; ${ }^{b}$ Currently at: Department of Chemistry, McGill University, 800 Sherbrooke St. W., Montreal, QC, Canada, H3A OB8; ${ }^{c}$ Department of Chemical \& Petroleum Engineering, Schulich School of Engineering, University of Calgary, 2500 University Dr NW, Calgary, AB, Canada T2N 1N4

E-mail: trudels@ucalgary.ca
}

Contents of the Supplementary Data

Figure S1 Photographs of Si(100) surfaces water wettability before and after etching

Figure S2 Photographs of Si(100) surfaces before and after blanket, in-solution thiol-ene reaction

Figure S3 SEM of negative control reaction 
Fig. S1. Image showing hydrophilic and hydrophobic character of silicon wafer before (left) and after (right) etching with ammonium fluoride.

Fig. S2. Image showing silicon wafer before (left) and after (right) a reaction done under blanket UV exposure, without a stamp. The reacted wafer is clearly matte.

Fig. S3. Negative control reaction sample, wherein no UV light or PDMS stamp was employed. Image shows no NPs bonded to surface. Ink contained $10 \mathrm{mg}$ NPs, $0.25 \mathrm{~g}$ DMPA, $1 \mathrm{~mL}$ solvent. The ene-terminated $\mathrm{Si}(100)$ was immersed in this ink for $1.5 \mathrm{hr}$. 